\title{
Inertial Impact Tests to Identify the Plastic Properties of Metals
}

\author{
Frances Davis ${ }^{1, *}$, Lloyd Fletcher $^{1}$, and Fabrice Pierron ${ }^{1}$ \\ ${ }^{1}$ Faculty of Engineering and the Environment, University of Southampton, Highfield Campus, SO17 1BJ, United Kingdom
}

\begin{abstract}
The behavior and failure mechanisms of materials often change at high strain rates $(>1001 / \mathrm{s})$ when compared with their quasi-static response. These differences are critical when designing structures or components that will be subjected to impact or blast loads. The recent progress in ultra-high speed imaging and full-field measurement techniques provides a unique opportunity to improve the quality of high strain rate test data. The objective of the current work is to design and validate an experimental technique to identify the elastoplastic material properties of metals. The methodology uses an ultra-high speed camera and the grid method to obtain time-resolved full-field deformation data as impact induced stress waves propagate in a sample. The virtual fields method is then used to inversely identify the plastic properties of the specimen. The results for five aluminum 6082-T6 impacted at $50 \mathrm{~m} / \mathrm{s}$ are presented.
\end{abstract}

\section{Introduction}

Numerous methods for characterizing the high strain rate response of metals have been proposed [1]; however, the split-Hopkinson bar (SHB) has been by far the most popular method to study material behavior at strain rates between $10^{3}$ to $10^{4} \mathrm{~s}^{-1}$. The split-Hopkinson bar relies on one-dimensional wave propagation theory to calculate the stress, strain, and strain rate in the specimen during the test. Notably the use of one-dimensional wave theory and point strain measurements (strain gauges) means that the stress and strain state in the sample is assumed to be uniaxial and uniform throughout the test. While SHB testing remains popular the possibilities for test configurations can be greatly expanded through the use of ultra-high speed cameras and inverse techniques - leading to the creation of new test methodologies. The inertial impact tests (Figure 1) proposed in this work rely on the use of an ultrahigh speed camera and full-field measurement techniques to image impact induced stress waves propagating within a specimen. By combining full-field deformation data with the virtual fields method investigators have identified the linear elastic [2], failure [3], and hyperelastic [4, 5] properties of composite plates, concrete, and rubbers, respectively.

The objective of the current work is to design and validate an experimental technique to identify the elastoplastic material properties of metals. First the virtual fields method will be introduced. Next, in order to design the experiments, explicit finite element analyses will be used to perform a parametric study on the various experimental options and identify several 'best case' experimental setups. Finally, the results of performing the 'best case' experiments are presented. The methodology uses an ultra-high speed camera and the grid method [6] to ob-

\footnotetext{
*e-mail: frances.davis@ soton.ac.uk
}

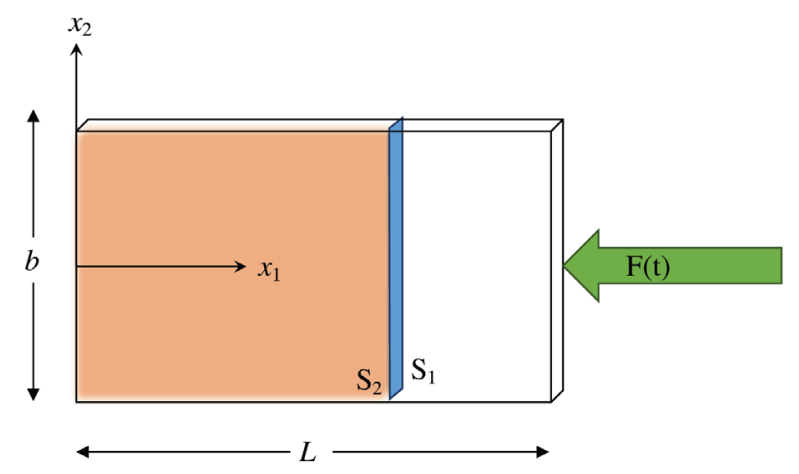

Fig. 1. Thin rectangular specimen loaded on the right hand side by a time varying force $\mathrm{F}(\mathrm{t})$ which denotes the impact load. The average stress over any vertical cross-section $S_{1}$ can be found using Eq. 2.

tain time-resolved full-field deformation data as impact induced stress waves propagate in the sample. The virtual fields method is then used to inversely identify the plastic properties of the specimen.

\section{Virtual Fields Method}

The principle of virtual work is derived from an integral form of equilibrium equations [7]. For the case presented in Figure 1, ignoring body forces the following equation is obtained :

$$
\overbrace{\int_{S_{f}} \boldsymbol{T} \cdot \boldsymbol{u}^{*} \mathrm{~d} S}^{W_{\text {ext }}^{*}}-\overbrace{\int_{V} \boldsymbol{\sigma}: \boldsymbol{\epsilon}^{*} \mathrm{~d} V}^{W_{i n t}^{*}}=\overbrace{\int_{V} \rho \boldsymbol{a} \cdot \boldsymbol{u}^{*} \mathrm{~d} V}^{W_{\text {acc }}^{*}}
$$

where $\boldsymbol{T}$ is the traction vector, $\boldsymbol{\sigma}$ is the stress tensor, $\boldsymbol{a}$ is the acceleration vector, $\rho$ is the density of the material, and 
$\boldsymbol{u}^{*}$ and $\boldsymbol{\epsilon}^{*}$ are the virtual displacement vector and associated virtual strain tensor, respectively. Assuming a state of plane stress and imposing a virtual displacement of $u_{1}^{*}=1$ and $u_{2}^{*}=0$, the first integral in Eq. 1 will equal the average stress in the $x_{1}$ direction. This set of virtual fields also cancels out the contribution of internal virtual work, $W_{\text {int }}$, leaving only the inertial virtual work, $W_{a c c}$. Replacing the integrals with discrete sums the following equation was found:

$$
{\overline{\sigma_{11}\left(x_{1}, t\right)}}^{S 1}=\rho x_{1}{\overline{a_{1}\left(x_{1}, t\right)}}^{S 2} .
$$

In Eq. 2, the overbars indicate averages over area specified in the superscript (Figure 1). By varying the value of $x_{1}$, the average stress for any transverse section can be identified from the acceleration. To identify the elasto-plastic material parameters, a set of non-linear equations is minimized. The cost function to be minimized is sum of the squared difference between the average stress in each section calculated from the acceleration (Eq. 2) and the average stress in each section calculated using the constitutive model. The identification is iterative; the cost function is evaluated for several different combinations of the material parameters, through the constitutive model, searching for the set of parameters that minimize the cost function. In this study, a linear elastic model with linear hardening was used to describe the elasto-plastic response requiring two parameters to be identified, the yield stress, $\sigma_{0}$, and hardening modulus, $H$.

\section{Experimental Design}

The impact of : (a) the length of the impactor, $(b)$ the length and height of the specimen, and $(c)$ the speed of the impactor were studied using an explicit finite element model (Abaqus Explicit, v. 6.13). In the proposed impact experiment, a cylindrical projectile impacts a thin rectangular specimen (Figure 1). Both the impactor and specimen were modeled in two dimensions using the assumption of plane stress. The specimen and impactor were both modeled as elasto-plastic, combining linear elasticity, the von Mises yield criterion, and a linear hardening model. The material parameters used to describe the impactor and specimens are $\mathrm{E}=70 \mathrm{GPa}, v=0.30, \rho=2700 \mathrm{~kg} / \mathrm{m}^{3}, \sigma_{0}$ $=350 \mathrm{MPa}, H=0.7 \mathrm{GPa}$ and $\mathrm{E}=205 \mathrm{GPa}, v=0.30$, $\rho=7850 \mathrm{~kg} / \mathrm{m}^{3}, \sigma_{0}=650 \mathrm{MPa}, H=2 \mathrm{GPa}$ for the aluminum specimen and steel impactor, respectively. Note that aluminum 6082-T6 was selected for the sample since previous studies have shown it is rate-independent over a wide range of strain rates at room temperature $[8,9]$. Modeling only rate-independent plasticity simplifies both the finite element model and the inverse identification. The duration of the simulation was set to $25.6 \mu$ s and the automatic timestep calculations of Abaqus were used. The displacement, acceleration, strain, and stress were output from the FE simulation at 128 equally spaced intervals of $0.2 \mu \mathrm{s}$. The format of the data was selected to mimic the frequency and number of images that can be collected using an Shimadzu HPV-X ultra high speed camera.

\subsection{Influence of impactor and specimen geometry}

The geometry of the impactor and specimen were varied to determine what combinations will produce the maximum pulse duration on the impact edge. A total of 9 models were generated (Table 1) varying the impactor length (Model 1 - 4), doubling the specimen length (Model 56 ), and varying the specimen height (Model 7-9). For all of the simulations an impact speed of $50 \mathrm{~m} / \mathrm{s}$ was used. In Table 1 , the maximum average stress $\overline{\sigma_{11}}$ on the impact edge, the percentage of the specimen length where the equivalent plastic strain, $\varepsilon_{p}$, is greater than $0.5 \%$, and the maximum equivalent plastic strain are given for each of the 9 geometric models. For the longer specimens (Model 5 and 6), although the length of the specimen has doubled only a field of view of $45 \mathrm{~mm}$ near impact edge would be imaged during an experiment, as a result, the effective percentage of the length that is deformed plastically is shown. While doubling the specimen and impactor length does increase the percentage of sample deformed and the maximum equivalent plastic strain, the improvements are not significant. Based on these observations the geometries where the length of the specimen matches the length of the impactor will be preferred.

\subsection{Influence of impactor speed}

The effect of impactor speed on the magnitude of the stress transmitted to the sample was explored; four impact speeds were simulated $25,50,100$, and $130 \mathrm{~m} / \mathrm{s}$. Table 1 shows that the magnitude of the pulse increased with increasing impactor velocity. For the lowest speed, the sample is not deformed plastically (Model 10). When the impact speed is doubled from 50 to $100 \mathrm{~m} / \mathrm{s}$ the percentage of the sample that is deformed plastically increased by nearly $15 \%$ while the magnitude of the equivalent plastic strain increased by a factor of 4 . At the higher impact speeds with plastic strain levels exceeding $10 \%$ there is likely to be crushing at the impact edge which would invalidate the plane stress assumption and propagate waves in three dimensions [10]. As a result, only an impact speed of $50 \mathrm{~m} / \mathrm{s}$ will be considered further.

\section{Validation of the VFM}

The stress gauge formula from Eq. 2 was validated on acceleration data calculated from the finite element displacement data. In Figure 2, the stresses obtained from acceleration are compared with the average FE stresses for four different transverse sections as a function of time for Model 2. In addition, the error between the finite element stresses and the stress gauge stresses, $\overline{\sigma_{11}} F E-\bar{\sigma}_{11} S G$, is also shown. In general the stresses reconstructed from acceleration follow the finite element stresses well, however the rise of the pulse, the stresses reconstructed from acceleration do not capture the sharp temporal gradient of stress well. The error is introduced by the numerical damping (linear bulk viscosity) applied in simulation. The damping 
Table 1. Geometry, impact speed, and degree of plastic deformation for each model.

\begin{tabular}{lccccccc} 
& $\begin{array}{c}L_{\text {impactor }} \\
\mathrm{mm}\end{array}$ & $\begin{array}{c}L_{\text {specimen }} \\
\mathrm{mm}\end{array}$ & $\begin{array}{c}h_{\text {specimen }} \\
\mathrm{mm}\end{array}$ & $\begin{array}{c}v_{0} \\
\mathrm{~m} / \mathrm{s}\end{array}$ & $\begin{array}{c}\bar{\sigma}_{11}^{\max } \\
\mathrm{MPa}\end{array}$ & $\begin{array}{c}\frac{L_{\text {plast }}}{45} \\
\%\end{array}$ & $\begin{array}{c}\varepsilon_{p}^{\text {max }} \\
\%\end{array}$ \\
\hline Model 1 & 22.5 & 45 & 32 & 50 & -413 & 20 & 3.53 \\
Model 2 & 45 & 45 & 32 & 50 & -413 & 22 & 4.25 \\
Model 3 & 67.5 & 45 & 32 & 50 & -413 & 22 & 4.27 \\
Model 4 & 90 & 45 & 32 & 50 & -413 & 22 & 4.27 \\
\hline Model 5 & 45 & 90 & 32 & 50 & -413 & 32 & 4.47 \\
Model 6 & 90 & 90 & 32 & 50 & -413 & 34 & 5.11 \\
\hline Model 7 & 45 & 45 & 30 & 50 & -413 & 22 & 4.45 \\
Model 8 & 45 & 45 & 28 & 50 & -413 & 22 & 4.72 \\
Model 9 & 45 & 45 & 26 & 50 & -413 & 22 & 5.07 \\
\hline Model 10 & 45 & 45 & 32 & 25 & -272 & 0 & 0 \\
Model 11 & 45 & 45 & 32 & 100 & -476 & 37 & 15.8 \\
Model 12 & 45 & 45 & 32 & 130 & -513 & 41 & 21.9 \\
\hline
\end{tabular}
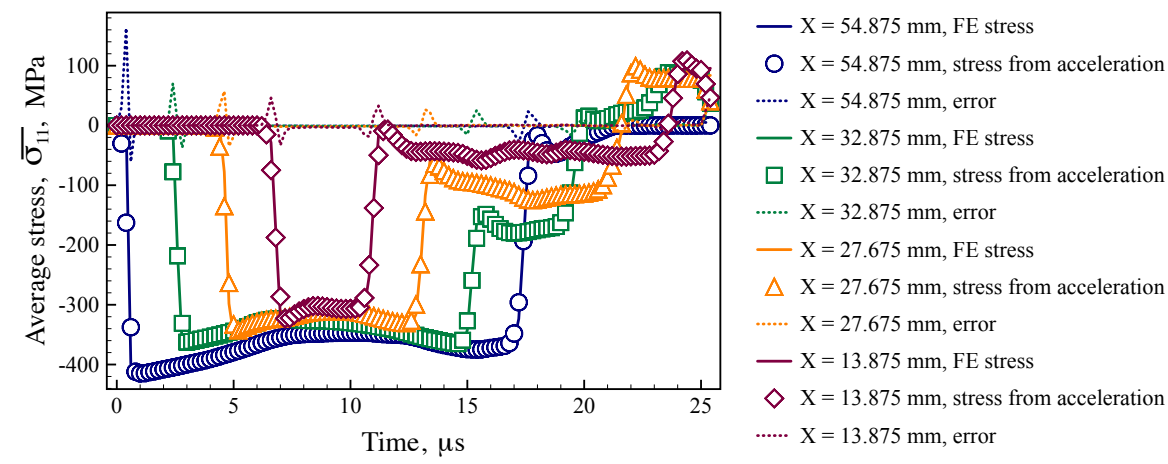

Fig. 2. Validation of the stress-gauge formulation for four different vertical cross-sections in the sample.

stress produced in the simulation is removed from the calculated element stresses in Abaqus however, the influence of the numerical damping is still present the FE displacement. In addition, in Abaqus Explicit the displacement is calculated from the acceleration over a single time step. The numerical differentiation used to calculate the acceleration from the displacement operates over 5 time steps and as a result the derivative calculation will further smear the acceleration.

The approach detailed in Sec. 2 was used to identify the yield stress and hardening modulus of the specimen from FE data. To mimic the experimental case only the FE displacements were used. Strain and acceleration were calculated from the displacement by spatial and double temporal differentiation, respectively. A return-mapping algorithm (RMA) based on the work of Sutton et al. [11] was implemented in MatLab (v. 9.0, R2016a). The RMA was used to calculate $\overline{\sigma_{11}}$ in Eq. 2 from the calculated strains. The plastic parameters, $\sigma_{0}$ and $H$, were identified by minimizing the cost function built from Eq. 2 using the function fmincon in MatLab. The stress gauge VFs are able to identify the yield stress, $\sigma_{y}=349.5 \mathrm{MPa}(0.14 \%)$ and hardening modulus, $H=623.1 \mathrm{MPa}(11 \%)$. Although the error on the hardening modulus is high, the absolute error between $\bar{\sigma}_{11}$ calculated using the model parameters

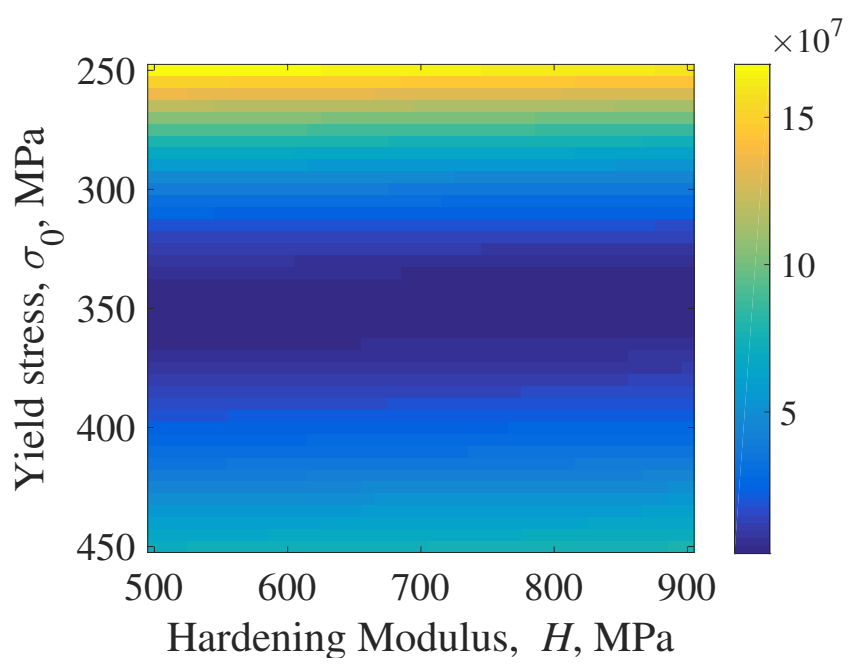

Fig. 3. Cost function map for a range of yield stress and hardening values for the aluminum sample.

input in the FE simulation and $\bar{\sigma}_{11}$ calculated using the identified model parameters is less than $4 \mathrm{MPa}$.

The same sources of error that effect the stress gauge formula, numerical damping and the derivative calculation, also impact the inverse identification. Figure 3 shows 
a map of the cost function that was obtained for the aluminum specimen impacted at $50 \mathrm{~m} / \mathrm{s}$ using the stress gauge virtual fields. Notice the valley formed indicating a limited sensitivity to the hardening modulus while there is a well defined gradient with respect to yield stress. This limited sensitivity leads to poorer identification of the hardening modulus.

\section{Inertial Impact Experiments}

A series of impact tests were performed using the gas gun facilities in the Testing Structures and Research Laboratory at the University of Southampton. Steel projectiles with a diameter of $50 \mathrm{~mm}$ and a length of $70 \mathrm{~mm}$ were fired by the gas gun to reach speeds of $50 \mathrm{~m} / \mathrm{s}$. Each projectile then impacts a steel waveguide which had the same dimensions as the projectile. A waveguide was used to mitigate the influence of any misalignment and to allow some homogenization of the stress wave before it reached the specimen. Five aluminum 6082-T6 samples $(b=43$ x $L=70$ x $4 \mathrm{~mm}$ ) were cut from a single sheet. First, a white rubber paint was sprayed on the sample surface and then black dots were printed on top to produce a white grid with a pitch of $0.9 \mathrm{~mm}$. An ultra-high speed camera (HPV-X, Shimadzu) was used to image the specimen. The HPX-X camera (10-bit, $400 \times 250$ px) was operated at 5 Mfps and captured 128 images for a total recording time of $25.6 \mu \mathrm{s}$. A copper make trigger between the projectile and the waveguide was used to start the camera. A flash was used to illuminate the field of view and was triggered by a series of light gates at the end of the gas gun barrel.

The grid method with a compensation [6] was used to calculate time-resolved displacement fields from the collected images and provides displacement values for every camera pixel. The displacement field was then temporally smoothed using a Savitzky-Golay filter (3rd order, 15 points). Then the acceleration was calculated by differentiating twice in time. The displacements were smoothed in space using a Gaussian filter with a window of 9 points before the strain was computed.

A total of five aluminum specimens were successfully tested at an impact speed of $50 \mathrm{~m} / \mathrm{s}$ which corresponds to a maximum strain rate of $\approx 1500 \mathrm{~s}^{-1}$. Eq. 2 can be used to generate stress-strain curves at any transverse section. Figure 4 shows one such representative stress-strain curve. A clear elastic region followed by yield can be observed. Note that the $\mathrm{x}$-position is measured from the free edge and this stress strain curve is for a section approximately $3 \mathrm{~mm}$ from the impact edge.

The average yield stress and hardening modulus identified from the tests were $298 \mathrm{MPa}$ and $1.6 \mathrm{GPa}$, respectively. For comparison, in house quasi-static tests identified a yield stress of $272 \mathrm{MPa}$ and hardening modulus of 1 $\mathrm{GPa}$ in tension. The average values identified in from the high strain rate test in compression are higher than those measured at quasi-static rates with the hardening modulus significantly increasing. However the previously identified error in the hardening modulus when processing finite element data means that the accuracy of the identified hard-

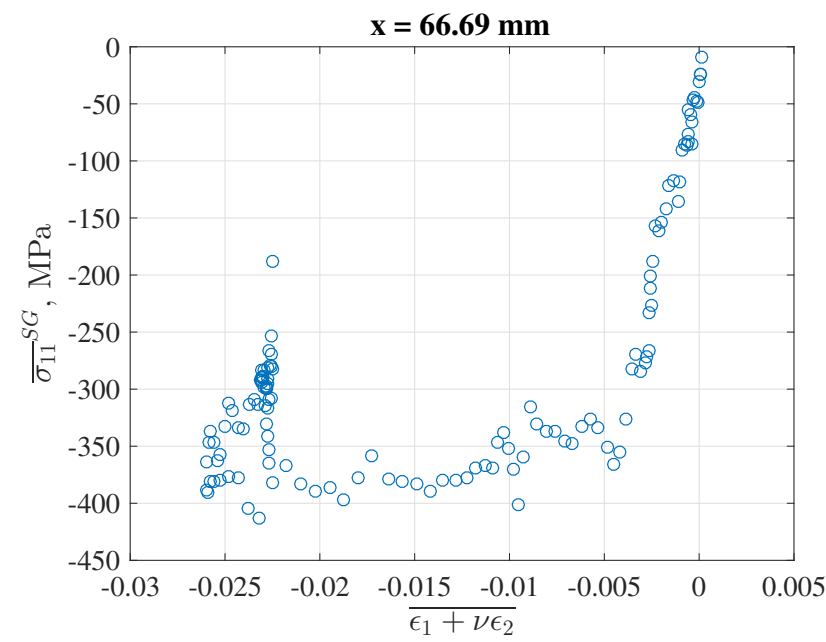

Fig. 4. Stress-strain curve at $x=66.69 \mathrm{~mm}$ for sample AL 4 . Note that a value of $v=0.3$ is always used for plotting even after the sample yields.

ening modulus is unknown. Currently a process of simulating the experimental chain with image deformation [12] is in process to better quantify the uncertainty in the identified yield stress and hardening modulus.

\section{Conclusions}

In this paper, a technique for identifying the plastic model parameters from an inertial impact test was presented. The results obtained using finite element data show that the stress gauge virtual fields are able to recover both the yield stress and hardening modulus. However by plotting the cost function, it was found that there is limited sensitivity to the hardening modulus when the stress gauge virtual fields are used. The experimental results, as expected, identify yield stresses at high strain rate which are only slightly higher than the quasi-static value. Additional work to simulate the entire experimental chain using image deformation is currently in process. Further, an experimental campaign is underway to characterize the more complex rate-dependent case of steel 316L.

\section{References}

1. J.E. Field, S.M. Walley, W.G. Proud, H.T. Goldrein, C.R. Siviour, International Journal of Impact Engineering 30, 725 (2004)

2. F. Pierron, H. Zhu, C.R. Siviour, Philosophical transactions of the Royal Society A 372, 20130195 (2014)

3. F. Pierron, P. Forquin, Strain 48, 388 (2012)

4. S.H.H. Yoon, I. Giannakopoulos, C.R. Siviour, International Journal of Solids and Structures 69-70, 553 (2015)

5. S.H.H. Yoon, M. Winters, C.R. Siviour, Experimental Mechanics 56, 25 (2016)

6. C. Badulescu, M. Grédiac, J.D. Mathias, Measurement Science and Technology 20, 095102 (2009) 
7. F. Pierron, M. Grédiac, The Virtual Fields Method (Springer New York, New York, NY, 2011), ISBN 978-1-4614-1823-8,

8. W. Moćko, J.A. Rodríguez-Martínez, Z.L. Kowalewski, A. Rusinek, Strain 48, 498 (2012)

9. X. Chen, Y. Peng, S. Peng, S. Yao, C. Chen, P. Xu, PLoS ONE 12, e0181983 (2017)
10. H. Zhu, F. Pierron, Experimental Mechanics 56, 3 (2016)

11. M.A. Sutton, X. Deng, J. Liu, L. Yang Experimental Mechanics36, 2 (1996)

12. B. Lukić, D. Saletti, P. Forquin, Philosophical transactions of the Royal Society A 375, 20160168 (2017) 
